Supplement of Hydrol. Earth Syst. Sci., 22, 4667-4683, 2018

https://doi.org/10.5194/hess-22-4667-2018-supplement

(c) Author(s) 2018. This work is distributed under

the Creative Commons Attribution 4.0 License.

(c) (1)

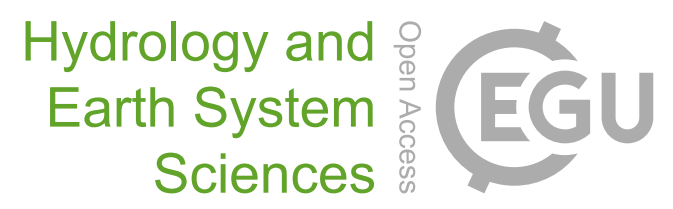

Supplement of

\title{
The potential of global reanalysis datasets in identifying flood events in Southern Africa
}

Gaby J. Gründemann et al.

Correspondence to: Gaby J. Gründemann (g.j.gruendemann@ @udelft.nl)

The copyright of individual parts of the supplement might differ from the CC BY 4.0 License. 
Supplement Table S1. River gauging stations in the Limpopo River basin that were selected in this research

\begin{tabular}{|c|c|c|c|c|}
\hline $\begin{array}{l}\text { Station } \\
\text { Number }\end{array}$ & River and station location & $\begin{array}{l}\text { Catchment } \\
\text { Area }\left[\mathrm{km}^{2}\right]\end{array}$ & Latitude & Longitude \\
\hline A2H006 & Pienaars River@ Klipdrift & 1028 & -25.380 & 28.317 \\
\hline A2H012 & Krokodil River@ Kalkheuwel & 2551 & -25.811 & 27.910 \\
\hline A2H013 & Magalies River @ Scheerpoort & 1171 & -25.777 & 27.761 \\
\hline A2H014 & Hennops River@ Skurweberg & 1007 & -25.798 & 27.985 \\
\hline A2H021 & Pienaars River @ Buffelspoort & 7483 & -25.129 & 27.629 \\
\hline A2H023 & Jukskei River@ Nietgedacht & 686 & -25.954 & 27.963 \\
\hline A2H024 & Brandvlei River@ Brandvlei & 13 & -26.151 & 27.595 \\
\hline A2H027 & Pienaars River @ Baviaanspoort & 357 & -25.664 & 28.351 \\
\hline A2H029 & Edendale Spruit @ Leeuwfontein & 129 & -25.647 & 28.390 \\
\hline A2H030 & Roodeplaat Spruit @ Louwsbaken se Loop Roodeplaat & 116 & -25.603 & 28.375 \\
\hline A2H032 & Selons River@ Moedwil & 522 & -25.638 & 27.027 \\
\hline A2H034 & Skeerpoort River@ Scheerpoort & 150 & -25.825 & 27.772 \\
\hline A2H036 & Koster River@ Steenbokfontein & 191 & -25.726 & 26.884 \\
\hline A2H039 & Waterkloof-Upper @ Rietvallei & 4 & -25.720 & 27.186 \\
\hline A2H044 & Jukskei River @ Vlakfontein & 798 & -25.896 & 27.935 \\
\hline A2H045 & Krokodil River@ Vlakfontein & 653 & -25.893 & 27.915 \\
\hline A2H047 & Little Jukskei River @ Klipfontein & 65 & -26.068 & 27.973 \\
\hline A2H048 & Krokodil River @ Krokodilpoort & 4691 & -25.573 & 27.754 \\
\hline A2H049 & Bloubank Spruit @ Riet Spruit Zwartkop & 371 & -25.977 & 27.836 \\
\hline A2H050 & Krokodil River@ Zwartkop & 148 & -25.991 & 27.842 \\
\hline A2H051 & Krokodil River@ Van Wyks Restant & 109 & -26.033 & 27.843 \\
\hline A2H053 & Sterkstroom@ Grootfontein & 88 & -25.809 & 27.476 \\
\hline A2H055 & Moretele River @ Derdepoort Pretoria & 106 & -25.692 & 28.293 \\
\hline A2H056 & Steenoond Spruit @ Belle Ombre Sta. & 10 & -25.735 & 28.179 \\
\hline A2H058 & Swart Spruit @ Rietfontein & 107 & -25.748 & 27.910 \\
\hline A2H059 & Krokodil River @ Vaalkop & 12674 & -25.206 & 27.558 \\
\hline A2H060 & Krokodil River@ Nooitgedacht & 20627 & -25.063 & 27.520 \\
\hline A2H061 & Apies River@ Rondavel & 625 & -25.471 & 28.264 \\
\hline
\end{tabular}




\begin{tabular}{|c|c|c|c|c|}
\hline A2H062 & Walker Spruit @ Sunnyside Pretoria & 8.1 & -25.759 & 28.220 \\
\hline A2H063 & Wonderboom Spruit @ Mayville Pretoria & 30 & -25.701 & 28.192 \\
\hline A2H099 & Buffels Spruit @ Roodepoort & 137 & -24.861 & 28.253 \\
\hline A4H002 & Mokolo River@ Zandrivier & 1777 & -24.286 & 28.096 \\
\hline A4H004 & Matlabas River@ Haarlem East & 1046 & -24.161 & 27.480 \\
\hline A4H005 & Mokolo River@ Dwaalhoek & 3786 & -24.081 & 27.773 \\
\hline A4H007 & Tambotie River @ Blakeney & 398 & -23.764 & 27.909 \\
\hline A5H004 & Palala River@ Muisvogelkraal & 629 & -23.982 & 28.400 \\
\hline A5H006 & Limpopo River@ Botswana & 98240 & -22.935 & 28.004 \\
\hline A6H006 & Little Nyl River@ Nylstroom & 168 & -24.699 & 28.407 \\
\hline A6H010 & Badseloop River@ Vischgat & 70 & -24.574 & 28.640 \\
\hline A6H011 & Great-Nyl River@ Modderpoort & 73 & -24.762 & 28.344 \\
\hline A6H012 & Olifant Spruit @ Olifantspoort & 120 & -24.665 & 28.476 \\
\hline A6H018 & Rasloop River @ Sussensvale & 12 & -24.771 & 28.349 \\
\hline A6H019 & Hessie se Water @ Rietspruit & 16 & -24.660 & 28.451 \\
\hline A6H020 & Middelfontein Spruit @ Middelfontein & 43 & -24.671 & 28.561 \\
\hline A6H021 & De Wet Spruit @ Groenvaley & 16 & -24.633 & 28.597 \\
\hline A6H024 & Kootjie se Loop Spruit @ Waterval & 23 & -24.317 & 28.916 \\
\hline A9H003 & Tshinane River @ Chibase & 62 & -22.898 & 30.524 \\
\hline A9H006 & Livhungwa River@ Barotta & 16 & -23.036 & 30.277 \\
\hline B1H002 & Spookspruit@ Elandspruit & 252 & -25.819 & 29.338 \\
\hline B1H004 & Klipspruit @ Zaaihoek & 376 & -25.674 & 29.173 \\
\hline B1H005 & Olifants River@ Wolwekrans & 3256 & -26.007 & 29.254 \\
\hline B1H012 & Little Olifants River @ Rondebosch & 1503 & -25.809 & 29.586 \\
\hline B2H003 & Bronkhorstspruit @ Bronkhorstspruit & 1574 & -25.799 & 28.736 \\
\hline B2H004 & Osspruit @ Boschkop & 123 & -25.925 & 28.586 \\
\hline В3H007 & Moses River@ Uitspanning & 971 & -25.272 & 29.181 \\
\hline B4H003 & Steelpoort River @ Buffelskloof & 2240 & -25.029 & 29.856 \\
\hline B4H005 & Waterval River@ Modderspruit & 188 & -25.036 & 30.219 \\
\hline B4H007 & Klein-Spekboom River @ Potloodspruit & 151 & -25.009 & 30.500 \\
\hline B4H009 & Dwars River @ Dwars River & 448 & -24.912 & 30.103 \\
\hline B4H010 & Dorps River @ Lydenburg Nat Res & 526 & -25.075 & 30.439 \\
\hline
\end{tabular}




\begin{tabular}{|l|l|c|l|l|}
\hline B6H001 & Blyde River @ Willemsoord & 518 & -24.680 & 30.802 \\
\hline B6H003 & Treur River @ Willemsoord & 92 & -24.687 & 30.814 \\
\hline B6H004 & Blyde River @ Chester & 2241 & -24.459 & 30.827 \\
\hline B6H005 & Blyde River @ Driehoek & 2204 & -24.518 & 30.832 \\
\hline B7H010 & Ngwabitsi River @ Harmony & 318 & -24.035 & 30.434 \\
\hline B7H013 & Mohlapitse River @ Mafefes Location & 263 & -24.173 & 30.103 \\
\hline B7H014 & Selati River @ Calais & 84 & -24.124 & 30.356 \\
\hline B8H009 & Great-Letaba River @ The Junction & 851 & -23.879 & 30.367 \\
\hline B8H010 & Letsitele River @ Mohlabas Location & 477 & -23.893 & 30.358 \\
\hline B8H011 & TSENDE & 441 & -23.527 & 31.403 \\
\hline B8H014 & Great-Letaba River @ Grysappel & 294 & -23.880 & 30.080 \\
\hline B8H017 & Great-Letaba River @ Prieska & 2619 & -23.647 & 30.718 \\
\hline B9H001 & Shisha River @ Vlakteplaas Kruger National Park & 648 & -22.839 & 31.237 \\
\hline B9H003 & Shingwidzi River @ Kanniedood Kruger National Park & 4540 & -23.143 & 31.463 \\
\hline E35 & Chokwe & 342000 & -24.508 & 33.008 \\
\hline
\end{tabular}


Supplement Table S2. Reported flood events identified in the Limpopo basin between 1979 and 2012, with the year of occurrence and the region affected (see Figure 1 for the definition of the regions)

\begin{tabular}{|c|c|c|c|c|c|c|c|c|c|}
\hline No. & Year & $\begin{array}{l}\text { Duration } \\
\text { [days] }\end{array}$ & BW & MZ & ZW & ZA1 & ZA2 & ZA3 & ZA4 \\
\hline 1 & 1981 & 26 & & TRUE & & & & & \\
\hline 2 & 1984 & 8 & & TRUE & & & & TRUE & TRUE \\
\hline 3 & 1985 & 7 & & TRUE & & & & & TRUE \\
\hline 4 & 1985 & 1 & & & & & TRUE & & \\
\hline 5 & 1987 & 1 & & & & & & & TRUE \\
\hline 6 & 1988 & 1 & TRUE & & TRUE & TRUE & & TRUE & TRUE \\
\hline 7 & 1988 & 5 & TRUE & TRUE & TRUE & & & TRUE & TRUE \\
\hline 8 & 1991 & 5 & & & & & & & TRUE \\
\hline 9 & 1992 & 1 & & TRUE & & & & & \\
\hline 10 & 1992 & 2 & & & TRUE & & & & \\
\hline 11 & 1992 & 3 & & & TRUE & & & & \\
\hline 12 & 1995 & 2 & & & & & & & TRUE \\
\hline 13 & 1995 & 1 & & & & & TRUE & & \\
\hline 14 & 1995 & 6 & TRUE & & & & & & \\
\hline 15 & 1995 & 6 & & & & & TRUE & & \\
\hline 16 & 1996 & 1 & & & & & TRUE & & \\
\hline 17 & 1996 & 3 & & TRUE & & & & & \\
\hline 18 & 1996 & 5 & & & & & & & TRUE \\
\hline 19 & 1996 & 6 & & & & TRUE & TRUE & TRUE & \\
\hline 20 & 1996 & 17 & & TRUE & & & & & \\
\hline 21 & 1997 & 1 & & & & & TRUE & & \\
\hline 22 & 1998 & 1 & & & TRUE & & & & \\
\hline 23 & 1999 & 8 & & TRUE & & & & & \\
\hline 24 & 2000 & 45 & TRUE & TRUE & TRUE & TRUE & TRUE & TRUE & TRUE \\
\hline 25 & 2000 & 3 & & & & & & TRUE & \\
\hline 26 & 2001 & 31 & & TRUE & & & & & \\
\hline 27 & 2001 & 1 & & & & & TRUE & & \\
\hline 28 & 2001 & 10 & & & TRUE & & & TRUE & \\
\hline
\end{tabular}




\begin{tabular}{|l|c|c|l|l|l|l|l|l|l|}
\hline 29 & 2002 & 2 & & & & & & TRUE & TRUE \\
\hline 30 & 2003 & 1 & & TRUE & & & & & \\
\hline 31 & 2003 & 12 & & TRUE & TRUE & & & & \\
\hline 32 & 2005 & 51 & & TRUE & & & & & \\
\hline 33 & 2005 & 4 & & & & & TRUE & & \\
\hline 34 & 2006 & 3 & & & & & TRUE & & \\
\hline 35 & 2006 & 7 & TRUE & & & & TRUE & & \\
\hline 36 & 2006 & 3 & & & & & TRUE & & \\
\hline 37 & 2007 & 48 & & & TRUE & & & & \\
\hline 38 & 2008 & 9 & & & & & & TRUE & TRUE \\
\hline 39 & 2009 & 7 & & TRUE & & & & & \\
\hline 40 & 2009 & 13 & TRUE & & & & & & \\
\hline 41 & 2009 & 1 & & & & & TRUE & & \\
\hline 42 & 2009 & 58 & TRUE & & TRUE & & & & \\
\hline 43 & 2009 & 4 & TRUE & & & & & & \\
\hline 44 & 2009 & 1 & TRUE & & & & & & \\
\hline 45 & 2010 & 3 & & & & TRUE & TRUE & & TRUE \\
\hline 46 & 2010 & 48 & & TRUE & TRUE & TRUE & TRUE & TRUE & TRUE \\
\hline 47 & 2011 & 1 & & TRUE & & & & & \\
\hline 48 & 2012 & 29 & & TRUE & & & & & \\
\hline
\end{tabular}

